\title{
Pregnancy Morbidities in Korean Patients with Takayasu Arteritis: A Monocentric Pilot Study
}

\author{
Jung Yoon Pyo ${ }^{1}$, Jason Jungsik Song ${ }^{1,2}$, Yong-Beom Park ${ }^{1,2}$, and Sang-Won Lee ${ }^{1,2}$ \\ ${ }^{1}$ Division of Rheumatology, Department of Internal Medicine, Yonsei University College of Medicine, Seoul; \\ ${ }^{2}$ Institute for Immunology and Immunological Diseases, Yonsei University College of Medicine, Seoul, Korea.
}

We investigated pregnancy morbidities in Korean patients with Takayasu arteritis (TA) in a single tertiary hospital as a pilot study. We retrospectively reviewed the medical records of 12 pregnancies in seven patients with TA. All patients were diagnosed with TA based on the 1990 American College of Rheumatology classification criteria. The medical records of patients were well-documented, allowing review of clinical data including pregnancy morbidities. The angiographic and Ishikawa classifications at diagnosis and TA activity at delivery were assessed. Of the 12 pregnancies, two pregnancies ended in spontaneous abortion (16.7\%), and one pregnancy $(8.3 \%)$ had therapeutic abortion at 9 weeks due to maternal morbidity. Among the remaining nine pregnancies, only one child was delivered via normal spontaneous vaginal delivery, and the remaining eight were delivered by Caesarean section. Two out of nine (22.2\%) neonates were born with low birth weight, and one of them was born at 30 weeks of gestation. The most common maternal complication was hypertension affecting 7/12 (58.3\%) pregnancies. Preeclampsia occurred in one pregnancy, and gestational diabetes mellitus (GDM) occurred in two pregnancies. At delivery, disease activity of TA was stable in all pregnancies, and glucocorticoids were administered in nine pregnancies. Live birth rate of pregnant Korean patients with TA was 75\%. Future studies are needed to reduce pregnancy-related complications.

Key Words: Arteritis, morbidity, pregnancy, vasculitis

Takayasu arteritis (TA) is a rare chronic vasculitis that affects the large-sized vessels, such as the aorta and its major branches, coronary arteries, and pulmonary arteries. In 1908, the first case of TA was reported in a 21-year-old woman with retinal arteriovenous anastomoses and absence of pulse on the upper extremity. ${ }^{1}$ TA is currently classified based on the American College of Rheumatology (ACR) 1990 criteria (1990 ACR criteria), which include the following six items: 1 ) age $\leq 40$ years at disease onset, 2) claudication of the extremities, 3) decreased pulsation of one or both brachial arteries, 4) difference of at least

Received: June 11, 2020 Revised: September 3, 2020

Accepted: September 3, 2020

Corresponding author: Sang-Won Lee, MD, PhD, Division of Rheumatology, Department of Internal Medicine, Yonsei University College of Medicine, 50-1 Yonsei-ro, Seodaemun-gu, Seoul 03722, Korea.

Tel: 82-2-2228-1984, Fax: 82-2-393-6884, E-mail: sangwonlee@yuhs.ac

-The authors have no potential conflicts of interest to disclose.

(C) Copyright: Yonsei University College of Medicine 2020

This is an Open Access article distributed under the terms of the Creative Commons Attribution Non-Commercial License (https://creativecommons.org/licenses/ by-nc/4.0) which permits unrestricted non-commercial use, distribution, and reproduction in any medium, provided the original work is properly cited.
$10 \mathrm{~mm} \mathrm{Hg}$ in systolic blood pressure between the arms, 5) bruit over one or both subclavian arteries or the abdominal aorta, and 6) arteriographic narrowing or occlusion of the entire aorta, its primary branches or large arteries in the proximal upper or lower extremities, excluding arteriosclerosis, fibromuscular dysplasia, or other causes. If more than three of these six items are met, a patient can be classified as TA. ${ }^{2}$

TA often occurs in young women, and most patients are classified before the age of 40 years. ${ }^{3,4}$ Hence, concerns over pregnancy morbidities, which may be induced by either TA itself or medications for its treatment, or both in patients with TA of the childbearing age, have been raised. In clinical practice, pregnancy morbidities, such as spontaneous abortion, intrauterine growth restriction (IUGR), preeclampsia or eclampsia, gestational diabetes mellitus (GDM), and preterm birth, have been reported in patients with TA. ${ }^{5}$ However, it remains unclear whether the rate and severity of pregnancy morbidities in patients with TA are higher than that of the general population..$^{6-8}$ Korea is one of the countries with the highest prevalence of TA worldwide. ${ }^{9}$ However, to date, no study has evaluated pregnancy morbidities of TA in Korea. Therefore, we investigated pregnancy mor- 
bidities in the Korean patients with TA in a single tertiary hospital as a pilot study.

We retrospectively reviewed the medical records of 58 pregnancies in 37 patients with TA. We excluded 46 pregnancies due to lack of delivery data, such as gestational age. Finally, 12 pregnancies in seven patients with TA were included in this study. All patients fulfilled the 1990 ACR criteria ${ }^{2}$ and were classified as TA at the Rheumatology Division of Yonsei University College of Medicine, Severance Hospital between January 1995 and December 2017. The medical records of all patients were well-documented, allowing to collect and review clinical and laboratory data and medical histories, particularly pregnancy morbidities. This study was approved by the Institutional Review Board (IRB) of Severance Hospital (4-2019-0699), and the need for written informed consent was waived by the approving IRB due to the retrospective nature of this study.

The angiographic classification of TA was defined based on the new classification proposed by Hata, et al. ${ }^{10}$ in 1996: Type I involves branches from aortic arch; Type IIa involves ascending aorta, aortic arch, and its branches; Type IIb involves ascending aorta, aortic arch, and thoracic descending aorta; Type III affects thoracic aorta, abdominal aorta, and/or renal arteries; Type IV affects only the abdominal aorta and/or renal arteries; and Type V involves combined features. The Ishikawa's ${ }^{11}$ classification of TA was also applied to patients with TA based on Ishikawa's severity criteria, which include the following three groups: Group 1 (patients with no complications), Group 2 (patients with one of following complications: Takayasu retinopathy, secondary hypertension, aortic regurgitation, or aortic or arterial aneurysm); Group 2 was subdivided into class 2a and $2 \mathrm{~b}$ by severity, and Group 3 (patients with two or more complications listed above).

Active disease was defined as a state with the new onset of two or more of the following features: 1) systemic features, such as fever or myalgia; 2) elevation of erythrocyte sedimentation rate or C-reactive protein level; 3 ) features of vascular ischemia or inflammation, such as claudication of extremities, diminished pulse, bruits, vascular pain, or asymmetric blood pressure; and 4) new vascular lesion(s) on imaging studies. ${ }^{12}$ Stable disease was defined as a state without the abovementioned features.

In this study, spontaneous abortion, therapeutic abortion, and fetal and maternal complications, such as preterm birth, IUGR, congenital anomaly, neonatal intensive care unit admission, preeclampsia or eclampsia, gestational hypertension, GDM, were considered as pregnancy morbidities. ${ }^{6}$

Continuous variables are presented as medians with interquartile ranges, and categorical variables are presented as numbers with percentages. Statistical analyses were performed using the Statistical Package for the Social Sciences software version 25 for Windows (IBM Corp., Armonk, NY, USA).

The median age at diagnosis of seven patients with TA was 27.0 years, and that at the time of delivery of 12 pregnancies was 32.0 years. Initial presentation, arterial involvement, angi- ographic and Ishikawa classification are shown in Table 1. The most frequently affected vessel was the common carotid artery (71.4\%), followed by descending aorta $(57.1 \%)$. All but one patients were diagnosed with TA before conception. All except one patient, who was diagnosed with TA after delivery, had stable disease activity at delivery. Glucocorticoids were maintained throughout the pregnancies in nine out of 12 cases with the daily dose within $10 \mathrm{mg}$ per day (prednisolone equivalent). Immunosuppressive agents, such as azathioprine and methotrexate, were used in eight out of 12 cases before conception,

Table 1. Disease Characteristics of 7 Patients at the Diagnosis of TA and 12 Cases of Pregnancies

\begin{tabular}{|c|c|}
\hline Variables & Values \\
\hline \multicolumn{2}{|l|}{ Seven patients with TA } \\
\hline Ages at diagnosis (yr) & $27.0(13.0)$ \\
\hline \multicolumn{2}{|l|}{ Clinical features at diagnosis } \\
\hline Bruit (subclavian or aorta) & $5(71.4)$ \\
\hline Blood pressure difference $>10$ mm Hg & $4(57.1)$ \\
\hline Decreased brachial pulse & $1(14.3)$ \\
\hline Claudication & $0(0)$ \\
\hline \multicolumn{2}{|l|}{ Involved vessels at diagnosis } \\
\hline Common carotid artery & $5(71.4)$ \\
\hline Descending aorta & $4(57.1)$ \\
\hline Subclavian artery & $2(28.6)$ \\
\hline Ascending aorta & $2(28.6)$ \\
\hline Aortic arch & $2(28.6)$ \\
\hline Abdominal aorta & $2(28.6)$ \\
\hline Renal artery & $2(28.6)$ \\
\hline Iliac artery & $0(0)$ \\
\hline Pulmonary artery & $1(14.3)$ \\
\hline \multicolumn{2}{|l|}{ Angiographic classification at diagnosis } \\
\hline I & $2(28.6)$ \\
\hline Ila & $0(0)$ \\
\hline Ilb & $2(28.6)$ \\
\hline III & $1(14.3)$ \\
\hline IV & $0(0)$ \\
\hline V & $2(28.6)$ \\
\hline \multicolumn{2}{|l|}{ Ishikawa classification at diagnosis } \\
\hline 1 & $1(14.3)$ \\
\hline $2 a$ & $5(71.4)$ \\
\hline $2 b$ & $0(0)$ \\
\hline 3 & $1(14.3)$ \\
\hline \multicolumn{2}{|l|}{ Twelve pregnancies } \\
\hline Age of delivery & $32.0(12.0)$ \\
\hline Maternal age $>35$ years at delivery & $5(41.7)$ \\
\hline Assisted by reproductive technology & $0(0)$ \\
\hline \multicolumn{2}{|l|}{ Previous abortion history } \\
\hline Spontaneous abortion & $2(16.7)$ \\
\hline Induced abortion & $2(16.7)$ \\
\hline
\end{tabular}

TA, Takayasu arteritis.

Values are expressed as a median (interquartile range) or $\mathrm{n}(\%)$. 
but withheld while planning pregnancy.

Of the 12 pregnancies, nine neonates were delivered alive; two pregnancies ended in spontaneous abortion (16.7\%) at 4 and 9 weeks of gestation, and one pregnancy ( $8.3 \%)$ underwent therapeutic abortion at 9 weeks of gestation due to maternal morbidity (Table 2 and 3). No antiphospholipid-related antibodies were detected in the patients experiencing spontaneous abortion.

Of the nine live neonates, the median gestational age at delivery was 38 weeks, and the median birth weight was $3060 \mathrm{~g}$. Among them, preterm birth before 37 weeks of gestation occurred in one (11.1\%). Three pregnancy morbidities occurred. One pregnancy developed GDM and preeclampsia, and the baby was born at 38 weeks of gestation with birth weight of $2200 \mathrm{~g}$ due to IUGR. Another pregnancy developed GMD, but delivered normal weight baby. The other pregnancy resulted in premature labor, and underwent emergency caesarean section at 30 weeks of gestation. That baby was born with birth weight of $1240 \mathrm{~g}$. Only one child (11.1\%) was delivered via normal spontaneous vaginal delivery, and the remaining eight (88.9\%) were delivered by Caesarean section.

Six out of 12 cases of pregnancy (50\%) had underlying hypertension before pregnancy, which was assumed to be related to TA. Among them, maternal blood pressure in one pregnancy was poorly controlled at delivery, but there were no maternal/ fetal complications.

Table 2. Pregnancy Outcome of TA

\begin{tabular}{lc}
\hline \multicolumn{1}{c}{ Pregnancy outcome } & Values \\
\hline Total pregnancies & 12 \\
Live births & $9(75.0)$ \\
\hline Gestational age at delivery (weeks) & $38.0(13.0)$ \\
C/sec & $8(88.9)$ \\
\hline NSVD & $1(11.1)$ \\
Spontaneous abortion & $2(16.7)$ \\
Termination of pregnancy & $1(8.3)$ \\
Fetal outcome & \\
\hline Birth weight (g) & $3060(260)$ \\
\hline Preterm birth <37 weeks gestation & $1 / 9(11.1)$ \\
\hline Intrauterine fetal demise & 0 \\
\hline Small for gestational age & $1 / 9(11.1)$ \\
\hline Congenital anomaly & $0(0)$ \\
\hline Fetal NICU admission & $2(16.7)$ \\
\hline Neonatal death & $0(0)$ \\
\hline Maternal complications & $1(8.3)$ \\
\hline Preeclampsia & $0(0)$ \\
\hline Gestational hypertension & $2(16.7)$ \\
\hline Gestational diabetes & $1(8.3)$ \\
\hline Pregestational diabetes & 0 \\
\hline Thyroid diseases & \\
\hline
\end{tabular}

TA, Takayasu arteritis; $\mathrm{C} / \mathrm{sec}$, Caesarean section; NSVD, normal spontaneous vaginal delivery; NICU, neonatal intensive care unit.

Values are expressed as a median (interquartile range) or $\mathrm{n}(\%)$.

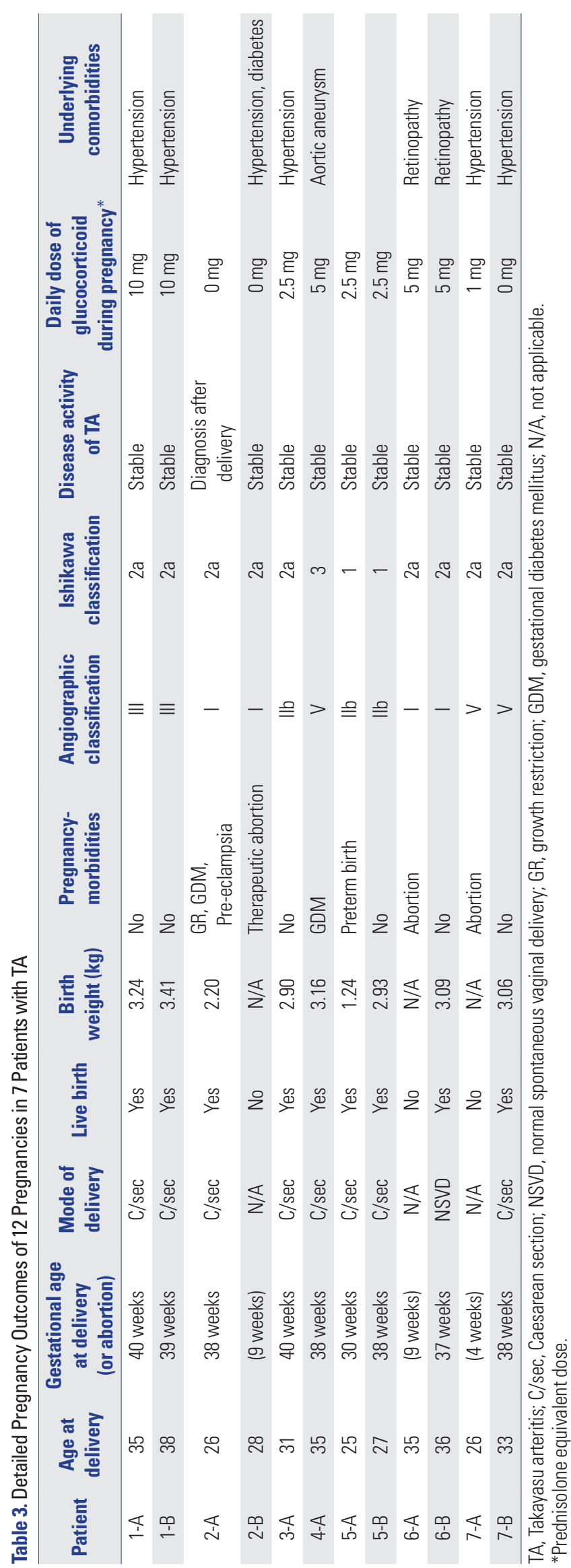

https://doi.org/10.3349/ymj.2020.61.11.970 
Among the eight cases of Caesarean section, only one case was planned for the surgery due to disease of the circulatory system associated with TA. Other indications for Caesarean section included advanced maternal age (2/8), arrest of descent $(1 / 8)$, minimal variability of fetal heart rate $(1 / 8)$, prior cesarean section (1/8), and unknown (2/8). Table 3 shows pregnancy outcomes for each of the 12 pregnancies in seven patients with TA, and Table 4 summarizes previous published reports and compares data with the current data.

To our knowledge, this is the first study to assess pregnancy morbidities in Korean patients with TA. In this study, successful delivery was observed in nine of 12 pregnancies (75.0\%) in a total of seven patients with TA. Three pregnancies had pregnancy morbidity: one pregnancy had preeclampsia, GDM, and IUGR; the other had GDM; and the last one was born prematurely.

Disease activity of TA remained stable at the time of delivery in all patients in our study. This observation was supported by a study demonstrating the influence of pregnancy on TA, which showed improving inflammatory markers during pregnancy in TA patients. ${ }^{13}$ Nonetheless, pregnancy morbidities were higher compared to the general population in Korea. We looked at the factors that caused this observation. First, we reviewed the angiographic classification. There were four pregnancies in angiographic classification Type I, three pregnancies in Type IIb, two pregnancies in Type III, and three pregnancies in Type V. Among pregnancies with Type I, two cases resulted in abortion (either therapeutic or spontaneous); one pregnancy was complicated with GDM, preeclampsia, and IUGR; and the other pregnancy resulted in normal delivery without complication. Among pregnancies with Type IIb, one pregnancy resulted in preterm birth, and the other two pregnancies resulted in normal delivery. Two pregnancies with Type III ended without complication; and among two Type $\mathrm{V}$ pregnancies, one pregnancy ended in spontaneous abortion, but the other pregnan- cy resulted in normal delivery. However, we could not conclude that Types I and V TA might be associated with pregnancy morbidities due to the small number of pregnancies in this study. Furthermore, we had assumed that the risk of gestational hypertension or preeclampsia may be theoretically increased in Types III, IV, and VTA, as renal arterial involvement in TA could increase the risk of renovascular hypertension; however, in our patients, preeclampsia was observed in only one patient with Type I TA. The absence of patients with Type IV TA made the interpretation of this association difficult. Second, the possibility of association between the Ishikawa classification of TA with pregnancy morbidities was assessed. There were two pregnancies with Ishikawa Type 1; one pregnancy ended in preterm birth, but the other resulted in normal delivery. There were nine pregnancies with Ishikawa classification Type 2a; and among them, three pregnancies ended in abortion (either therapeutic or spontaneous), and one pregnancy had pregnancy morbidity (GDM, preeclampsia, and IUGR). Remaining five pregnancies with Type 2a resulted in normal delivery. One pregnancy with Type 3 was complicated with GDM, but delivered normal weight neonate. Therefore, although the clinical significance is not strong, we suggest that attention should be paid to patients with Types I and V and Ishikawa Groups 2 and $3 \mathrm{TA}$. Lastly, the disease activity of TA remained stable at the time of delivery in all patients. This suggests two things. First, maintaining stable TA through proper steroid administration would not have resulted in serious complications, such as maternal death or TA aggravation. Second, the disease courses of $\mathrm{TA}$, including established arterial stenosis or aneurysm, did not seem to directly affect pregnancy morbidities. Therefore, we suggest that physicians should not be negative for steroid use and should make efforts to maintain low TA activity during pregnancy.

We searched for previous studies investigating the pregnan-

Table 4. Comparison of Pregnancy-Related Morbidities among the Previous and Present Studies

\begin{tabular}{|c|c|c|c|c|c|c|c|}
\hline $\begin{array}{c}\text { Reference } \\
\text { number (year) }\end{array}$ & Countries & $\begin{array}{l}\text { Time of pregnancy } \\
\text { based on diagnosis }\end{array}$ & $\begin{array}{c}\text { Number of } \\
\text { patients }\end{array}$ & $\begin{array}{l}\text { Number of } \\
\text { pregnancy }\end{array}$ & $\begin{array}{c}\text { Age at } \\
\text { pregnancy }\end{array}$ & $\begin{array}{l}\text { Live birth } \\
\text { [n, (\%)] }\end{array}$ & Pregnancy morbidities (\%) \\
\hline $14(2010)$ & India & After diagnosis & 15 & 37 & $27.6(4.7)^{*}$ & $25(67.6)$ & $\begin{array}{l}\text { GR (16.2), preterm birth (16.2), } \\
\text { preeclampsia (59.5) }\end{array}$ \\
\hline 7 (2012) & Japan & After diagnosis & 10 & 26 & $30.0(6.0)$ & $18(69.2)$ & GR (7.7), preterm birth (7.7) \\
\hline $15(2015)$ & Brazil & $\begin{array}{l}\text { Before diagnosis } \\
\text { After diagnosis }\end{array}$ & 89 & $\begin{array}{r}118 \\
38\end{array}$ & $25.1(5.2)^{*}$ & $\begin{array}{r}101(85.6) \\
35(92.1)\end{array}$ & $\begin{array}{l}\text { GR (16.8), preterm birth (11.9), eclampsia (2.5) } \\
\text { GR (34.2), preterm birth (45.7) }\end{array}$ \\
\hline $6(2015)$ & France & After diagnosis & 52 & 98 & $27.0(7.0)$ & $86(87.8)$ & GR (5.1), preterm birth (8.2) \\
\hline 16 (2017) & China & After diagnosis & 13 & 13 & $29.0(5.0)$ & $11(84.6)$ & $\begin{array}{l}\text { GR (15.4 preterm birth (15.4), } \\
\text { preeclampsia (15.4) }\end{array}$ \\
\hline $17(2017)$ & Norway & $\begin{array}{l}\text { Before diagnosis } \\
\text { After diagnosis }\end{array}$ & $\begin{array}{l}33 \\
23\end{array}$ & $\begin{array}{l}73 \\
37\end{array}$ & $\begin{array}{l}25.7(4.6)^{*} \\
29.4(4.0)^{*}\end{array}$ & $\begin{array}{l}51(69.9) \\
25(67.6)\end{array}$ & $\begin{array}{l}\text { Preeclampsia (9.1) } \\
\text { Preeclampsia (4.3), eclampsia (4.3) }\end{array}$ \\
\hline 18 (2019) & Turkey & After diagnosis & 11 & 22 & $31.0(7.0)$ & $16(72.7)$ & GR (13.6), preterm birth (18.2) \\
\hline Present study (N/A) & Korea & $\begin{array}{l}\text { After diagnosis except } \\
\text { for one }\end{array}$ & 7 & 12 & $32.0(12.0)$ & $9(75.0)$ & $\begin{array}{l}\text { GR (8.3), preterm birth (8.3), } \\
\text { preeclampsia (8.3), GDM (16.6) }\end{array}$ \\
\hline
\end{tabular}

GR, growth restriction; GDM, gestational diabetes mellitus.

Age at pregnancy are expressed as median (interquartile range) or * mean (standard deviation). 
cy outcomes in patients with TA, and summarized their findings in Table $4 .^{6,7,14-18}$ The median maternal age in this study was 32.0 years, which was higher than the median or mean maternal ages in the previous studies (range: 25.1 to 31.0 years). $\mathrm{Al}$ though our results are limited by the small sample size of this study, it could be explained by the socioeconomic aspect considering that the mean age of conception was the highest in Korea among the Organization for Economic Cooperation and Development (OECD) nations. ${ }^{19}$ In this study, the live birth rate of all pregnancies was $75 \%$, which was not significantly higher than that in previous studies. The highest age at conception of Korean patients may partially explain this result. However, it may be impossible to directly compare pregnancy morbidities between this study and the previous ones, due to varying study methods and medical services of different countries.

Our study had several limitations. The number of pregnancies in this study was too small to represent pregnancy morbidities in all Korean patients with TA. In particular, the retrospective design of this study resulted in a small number of available pregnancies due to missing data on the pregnancy and delivery outcomes in a considerable number of patients with TA. However, this is this first study to investigate pregnancy morbidities in Korean patients with TA. Since Korea is one of the countries with the highest prevalence of TA worldwide, it may be meaningful to describe and provide ethnic information on pregnancy morbidities in patients with TA in Korea as a pilot study. Therefore, a future study with a larger number of patients with TA may provide reliable information. In conclusion, the live birth rate of Korean patients with TA was $75 \%$. Pregnancy morbidities might be associated with the angiographic and Ishikawa classifications of TA, but further studies are needed for confirmation. Maintaining stable disease during pregnancy might be important to prevent serious maternal complications or TA progression.

\section{ACKNOWLEDGEMENTS}

This research was supported by Basic Science Research Program through the National Research Foundation of Korea (NRF) funded by the Ministry of Education (2017R1D1A1B03029050) and a grant from the Korea Health Technology R\&D Project through the Korea Health Industry Development Institute funded by the Ministry of Health and Welfare, Republic of Korea (HI14C1324).

\section{AUTHOR CONTRIBUTIONS}

Conceptualization: all authors. Data curation: Jung Yoon Pyo. Formal analysis: Jung Yoon Pyo and Sang-Won Lee. Funding acquisition: Sang-Won Lee. Investigation: Jung Yoon Pyo and Sang-Won Lee. Methodology: Jason Jungsik Song and Yong-Beom Park. Project administration: Sang-Won Lee. Resources: Jung Yoon Pyo and SangWon Lee. Software: Jung Yoon Pyo. Supervision: Jason Jungsik Song and Yong-Beom Park. Validation: Jason Jungsik Song and Yong-Beom
Park. Visualization: Jung Yoon Pyo and Sang-Won Lee. Writingoriginal draft: Jung Yoon Pyo. Writing-review \& editing: Sang-Won Lee. Approval of final manuscript: all authors.

\section{ORCID iDs}

Jung Yoon Pyo https://orcid.org/0000-0002-1866-6885 Jason Jungsik Song https://orcid.org/0000-0003-0662-7704 Yong-Beom Park https://orcid.org/0000-0003-4695-8620 Sang-Won Lee https://orcid.org/0000-0002-8038-3341

\section{REFERENCES}

1. Takayasu M. A case with peculiar changes of the retinal central vessels. Acta Soc Ophthalmol Jpn 1908;12:554-5.

2. Arend WP, Michel BA, Bloch DA, Hunder GG, Calabrese LH, Edworthy SM, et al. The American College of Rheumatology 1990 criteria for the classification of Takayasu arteritis. Arthritis Rheum 1990;33:1129-34.

3. Lucena AJ, Carvalho AC, Souza JA, Moron AF, Sun SY, Born D. Pregnancy follow-up and outcome in a patient with Takayasu's arteritis. Arq Bras Cardiol 2008;90:e33-5.

4. Mirault T, Messas E. Takayasu arteritis. Rev Med Interne 2016;37: 223-9.

5. Hauenstein E, Frank H, Bauer JS, Schneider KT, Fischer T. Takayasu's arteritis in pregnancy: review of literature and discussion. J Perinat Med 2010;38:55-62.

6. Comarmond C, Mirault T, Biard L, Nizard J, Lambert M, Wechsler B, et al. Takayasu arteritis and pregnancy. Arthritis Rheumatol 2015;67:3262-9.

7. Hidaka N, Yamanaka Y, Fujita Y, Fukushima K, Wake N. Clinical manifestations of pregnancy in patients with Takayasu arteritis: experience from a single tertiary center. Arch Gynecol Obstet 2012; 285:377-85.

8. Mohammad AJ, Mandl T. Takayasu arteritis in southern Sweden. J Rheumatol 2015;42:853-8.

9. Park SJ, Kim HJ, Park H, Hann HJ, Kim KH, Han S, et al. Incidence, prevalence, mortality and causes of death in Takayasu Arteritis in Korea - A nationwide, population-based study. Int J Cardiol 2017; 235:100-4

10. Hata A, Noda M, Moriwaki R, Numano F. Angiographic findings of Takayasu arteritis: new classification. Int J Cardiol 1996;54 Suppl: S155-63.

11. Ishikawa K. Natural history and classification of occlusive thromboaortopathy (Takayasu's disease). Circulation 1978;57:27-35.

12. Kerr GS, Hallahan CW, Giordano J, Leavitt RY, Fauci AS, Rottem M, et al. Takayasu arteritis. Ann Intern Med 1994;120:919-29.

13. Matsumura A, Moriwaki R, Numano F. Pregnancy in Takayasu arteritis from the view of internal medicine. Heart Vessels Suppl 1992;7:120-4.

14. Suri V, Aggarwal N, Keepanasseril A, Chopra S, Vijayvergiya R, Jain S. Pregnancy and Takayasu arteritis: a single centre experience from North India. J Obstet Gynaecol Res 2010;36:519-24.

15. Assad AP, da Silva TF, Bonfa E, Pereira RM. Maternal and neonatal outcomes in 89 patients with Takayasu arteritis (TA): comparison before and after the TA diagnosis. J Rheumatol 2015;42:1861-4.

16. Zhang Y, Li Y, Zhang J. Clinical analysis: 13 cases of pregnancy complicated with Takayasu arteritis. Ginekol Pol 2017;88:654-61.

17. Gudbrandsson B, Wallenius M, Garen T, Henriksen T, Molberg Ø, Palm Ø. Takayasu arteritis and pregnancy: a population-based study on outcomes and mother/child-related concerns. Arthritis Care Res (Hoboken) 2017;69:1384-90. 
18. Tanacan A, Unal C, Yucesoy HM, Duru SA, Beksac MS. Management and evaluation of pregnant women with Takayasu arteritis. Arch Gynecol Obstet 2019;299:79-88.

19. Organization for Economic Cooperation and Development. Age of mothers at childbirth and age-specific fertility (OECD Family Database 2019) [accessed on 2019 September 19]. Available at: https://www.oecd.org/els/soc/SF_2_3_Age_mothers_childbirth. pdf. 